\title{
Characterization of atrial histopathological and electrophysiological changes in a mouse model of aging
}

\author{
TAO LUO ${ }^{1,2^{*}}$, CUI-XIAN CHANG ${ }^{2 *}$, XIN ZHOU ${ }^{2}$, SHI-KUI GU ${ }^{2}$, TIE-MING JIANG ${ }^{2}$ and YU-MING LI $^{2}$ \\ ${ }^{1}$ Graduate School of Medicine, Tianjin Medical University; ${ }^{2}$ Institute of Cardiovascular Disease and Heart Center, \\ Pingjin Hospital, Logistics University of Chinese People's Armed Police Forces, Tianjin, P.R. China
}

Received August 13, 2012; Accepted October 4, 2012

DOI: $10.3892 / \mathrm{ijmm} .2012 .1174$

\begin{abstract}
The detailed mechanisms of age-related atrial structural and electrophysiological changes remain elusive. Small animal models have recently been used for the investigation of atrial tachyarrhythmia. In this study, we investigated the hypothesis that atrial structural and electrical characterization with aging provides a substrate for atrial fibrillation using a mouse model of aging. Male Kunming mice aged 2 (young), 12 (middle-aged) and 24 months (aged) were used in this study. A surface electrocardiogram and sinus node recovery time (SNRT) were recorded at baseline. Atrial fibrillation (AF) inducibility and duration were measured by a transesophageal electrode catheter. Collagen content was assessed by the collagen volume fraction. Whole cell configuration using the patch clamp technique was performed for the transient outward potassium $\left(I_{t o}\right)$ and ultra-rapid delayed rectifier potassium $\left(I_{k u r}\right)$ currents. P-wave duration, SNRT and rate-corrected SNRT were longer in the aged group than in the remaining 2 groups, paralleled by inducibility significantly being increased in the aged group. The right atrium had significantly higher levels of fibrosis than the left atrium in all the groups $(\mathrm{P}<0.05)$, whereas the extent of fibrosis in the left atrium had a higher positive correlation with age relative to the right atrium $(\mathrm{P}<0.05)$. Moreover, in old age, the dispersion of left relative to right atrium repolarization and augmented $I_{t o}$ currents contributed to vulnerability to AF. Nevertheless, $I_{k u r}$ currents in the atrial myocytes showed no age-related changes. The present study demonstrates that in addition to the structural alterations, aging can also cause integrative and cellular electrophysiological changes in a mouse model of aging, facilitating AF initiation and maintenance.
\end{abstract}

Correspondence to: Professor Yu-Ming Li, Institute of Cardiovascular Disease and Heart Center, Pingjin Hospital, Logistics University of Chinese People's Armed Police Forces, 220 Cheng-Lin Road, Tianjin 300162, P.R. China

E-mail: cardiolab@gmail.com

*Contributed equally

Key words: aging, atrium, atrial fibrillation, mouse

\section{Introduction}

Atrial fibrillation (AF), as a rhythm disorder characterized by chaotic electrical activity of the atria, is a frequent cause of cardiovascular morbidity and mortality worldwide. Its prevalence is 0.4 to $1.0 \%$ in the general population and increases with age (1). The mechanisms underlying AF remain elusive, and $\mathrm{AF}$ is thought to be maintained either via ectopic foci, multiple wavelets, or fibrillatory conduction emanating from a small number of stable rotors (2). Electrical and structural remodeling have emerged as crucial components in the persistence of AF (3). Electrical remodeling, such as changes in major repolarized ion channels, leads to the shortening of the action potential duration (APD) and the loss of APD rate-dependent adaptation, while structural remodeling (fibrosis) leads to an increase in atrial conduction slowing, re-entry, and thereby inducible AF (3).

Studies using large animal models (rabbits, dogs, canines and goats) have demonstrated a possible link between agerelated changes in histopathological and electrophysiological properties and the vulnerability to AF (4-8). However, timesaving and low-cost animal models, such as rats and mice, have not been fully characterized to date. Rodent models for AF have been reported in a limited number of studies, but there are insufficient data for young vs. old rats (9), young vs. middle-aged rats (10) or mice (11). Moreover, small animal models, in particular mouse models, provide powerful tools in the investigation of basic structural and electrical mechanisms of cardiac arrhythmias. It is believed that sustained fibrillatory activity does not exclusively depend on critical myocardial mass or that critical mass is smaller than the mouse atrial surface of $<35 \mathrm{~mm}^{2}$ (12). The inducibility of AF in the whole mouse heart in vivo has previously been evaluated using transvenous atrial stimulation, isolated stimulation and vagal activation by pharmacological intervention (29). Transesophageal stimulation is a minimally invasive method for atrial stimulation without direct atrial manipulation or mechanical changes during cardiac surgery or catheterization. Moreover, serial examinations of the same animal under different experimental conditions can be easily performed. These can facilitate the characterization of age-dependent electrophysiological properties in an individual animal.

Therefore, in the present study, adopting 3 sequential timepoints (young, middle-aged and aged) for further investigation in a mouse model, we aimed to evaluate dynamic alterations 
associated with atrial fibrosis, and to determine atrial electrical properties related to vulnerability to AF.

\section{Materials and methods}

Animals. For our study, male healthy Kunming mice aged 2 (young), 12 (middle-aged), and 24 months (aged) were obtained from the Laboratory Animal Center of the Academy of Military Medical Sciences (Beijing, China) and received humane care in compliance with the National Institutes of Health Guide for the Care and Use of Laboratory Animals (NIH publication no. 85-23, revised in 1996). We chose these ages of mice based on previous study (13). The ages of 2, 12 and 24 months are equivalent to the human age of $\sim 20,40$ and 70 years, respectively. The characteristics of the mice are shown in Table I.

In vivo global electrophysiological analysis. Sinus cycle length (SCL) was determined by averaging 3 consecutive R-R intervals. P-wave duration (PWD) was measured by determining the earliest onset and latest offset of atrial deflection from 3 simultaneously recorded surface leads.

For the transesophageal electrophysiological study, a 2-French octapolar mouse electrophysiological catheter [8 $0.5 \mathrm{~mm}$ circular electrodes; electrode pair spacing, $0.5 \mathrm{~mm}$ (Ciber Mouse; NuMed Inc., New York, NY)] was used for recording cardiac electrograms as well as for pacing the heart using consecutive electrode pairs. This catheter was inserted into the esophagus with a depth of 3-4 mm and unipolar recordings were obtained from each ring electrode. The threshold of pacing was examined by a $1 \mathrm{msec}$ pulse width and a pacing rate 10 beats/min faster than normal. The electrode catheter was adaptively positioned to the site closest to the left atrium to ensure that constant atrial capture and correspondence to the minimum threshold $(<1.5 \mathrm{~mA})$ were attained. Sinus node recovery time (SNRT) was measured after a $30-\mathrm{sec}$ pacing train with a basic cycle length (BCL) of $100 \mathrm{msec}$, a stimulus amplitude of 2-fold diastolic capture threshold and a stimulus duration of $1 \mathrm{msec}$. The SNRT was defined as the interval between the last stimulus in the pacing train and the onset of the first sinus return beat. Rate-corrected SNRT (CSNRT) was defined as the SCL subtracted from the SNRT.

Inducibility of atrial tachycardia and fibrillation (AT/F) was examined by applying 15 -sec bursts (25 msec BCL, 2-fold diastolic capture threshold and $1 \mathrm{msec}$ duration). This series of bursts was repeated 10 times. AF was defined as a period of rapid and fragmented atrial electrograms with irregular $\mathrm{AV}$-nodal conduction and ventricular rhythm for at least $1 \mathrm{sec}$ (14). AT was defined as rapid and regular rhythm lasting for at least $30 \mathrm{sec}$.

Histopathological analysis. After in vivo electrophysiological analysis, the mice were sacrificed and prepared for histopathological analysis. The aortas were then perfused via the common iliac bifurcation with pre-cooled PBS for $5 \mathrm{~min}$. The left and right auricles were quickly cut and snap-frozen in liquid nitrogen for $1 \mathrm{~min}$ and stored at $-80^{\circ} \mathrm{C}$. Sections $(5 \mu \mathrm{m}$ in thickness) of the paraffin-embedded tissue were stained with picrosirius red (Direct Red 80; Sigma Aldrich) (15). Briefly, tissue sections were rinsed in distilled water and incubated in saturated $0.1 \%$ picrosirius red for $90 \mathrm{~min}$. The sections were
Table I. Age and weight of the mice in the 3 groups.

\begin{tabular}{lccc}
\hline & Young & Middle-aged & Aged \\
\hline Age (months) & 2 & 12 & 24 \\
Weight $(\mathrm{g})$ & $35.4 \pm 4.2$ & $52.1 \pm 5.4$ & $60.5 \pm 7.2$ \\
\hline
\end{tabular}

Weight is presented as mean $\pm \mathrm{SD}$.

then rinsed twice with $0.01 \mathrm{~N} \mathrm{HCl}$ for $1 \mathrm{~min}$, dehydrated with an ethanol gradient and prepared for collagen analysis. Images were captured by a digital charge-coupled device (CCD) camera (Pro 150ES; Pixera Corporation, Los Gatos, CA), and connected to a polarized microscope (E600POL; Nikon, Tokyo, Japan) equipped and modified with a commercially available circular polarizer (Kenko, Tokyo, Japan). The collagen volume fraction (CVF) was calculated as described previously (15).

Patch-clamp experiments. To isolate high-quality mouse atrial myocytes, we adopted a Langendorff-perfused method based on modified intubation skills (16). In brief, mice were anesthetized using an intraperitoneal injection of $0.4 \%$ pentobarbital sodium solution at a dose of $50 \mathrm{mg} / \mathrm{kg}$ body weight. The inferior vena cava was exposed under direct vision following an abdominal median incision. While $4^{\circ} \mathrm{C}$ heparinized saline at a dose of 4-5 $\mathrm{ml}(100 \mathrm{U} / \mathrm{ml})$ was administrated into the inferior vena cava, the abdominal aorta was dissected for bloodletting. Subsequently, the thoracic aorta, isolated through thymus dissection following a left thoracotomy between the second and fourth intercostal space, was slightly intubated by a self-made catheter $(7 \mathrm{~F}$ paracentetic needle with blunt tip, ring-shaped notching $0.5 \mathrm{~mm}$ from the distal ending) at a position of $2 \mathrm{~mm}$ distance from the root of the aorta. A syringe with $3 \mathrm{ml}$ saline was connected to the end of the catheter for excluding the internal air. The successful intubation was fixed to the aorta by sealing with 5-0 silk. The heart was then quickly excised and removed to the Langendorff apparatus for immediate retrograde perfusion. Atrial myocytes were prepared using enzymatic digestion and then separated from Kraft-Brühe (KB) solution by centrifuging at $600 \mathrm{rpm}$ for $3 \mathrm{~min}$. The cells were maintained at room temperature for the experiments that followed and used within $10 \mathrm{~h}$.

Myocytes were transferred into an experimental chamber $(\sim 3 \mathrm{ml})$ mounted on the stage of an inverted microscope (TE2000-S; Nikon) and allowed to adhere to the glass bottom of the chamber. Cells were perfused with extracellular solution. A ruptured-patch whole cell voltage clamp was used to measure transient outward potassium $\left(I_{t o}\right)$ and ultra-rapid delayed rectifier potassium $\left(I_{k u r}\right)$ currents, and membrane capacitance $(\mathrm{Cm})$, while a current clamp was used for measuring a single cell action potential (AP). For $I_{t o}$ and $I_{k u r}$ measurements, the internal solution contained (in mmol/l) $110 \mathrm{~K}$-aspartate, $20 \mathrm{KCl}, 1 \mathrm{MgCl}_{2}, 5 \mathrm{Mg}$-ATP, $10 \mathrm{HEPES}$, 10 EGTA and $5 \mathrm{Na}_{2}$-creatine phosphate, $\mathrm{pH}$ 7.3-7.4 (NaOH). To block $\mathrm{Ca}^{2+}$-activated chloride currents, $0.01 \mathrm{mmol} / 1$ niflumic acid was added to the pipette solution. The external 
A

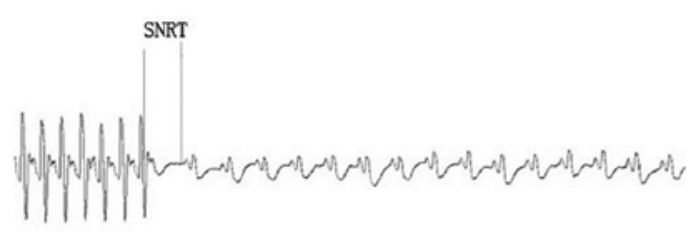

C

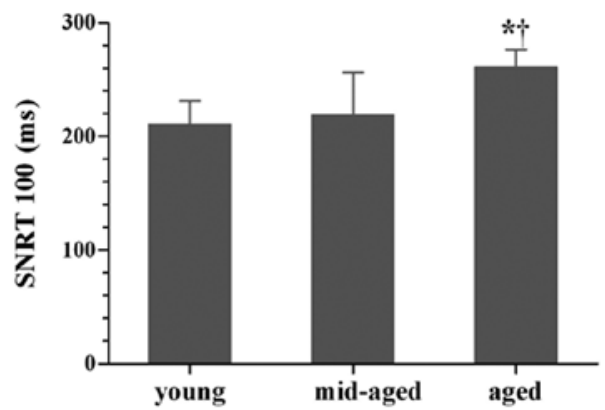

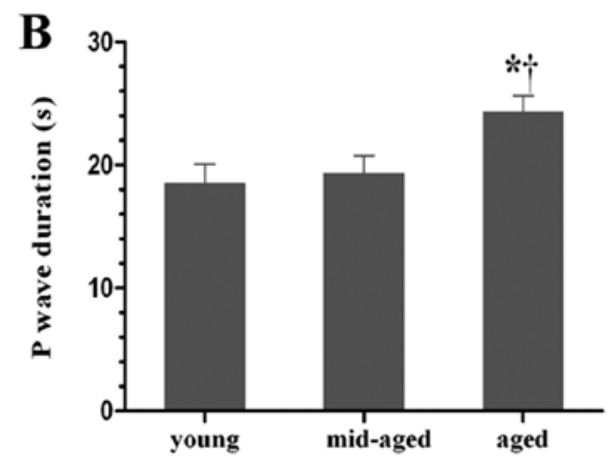

D

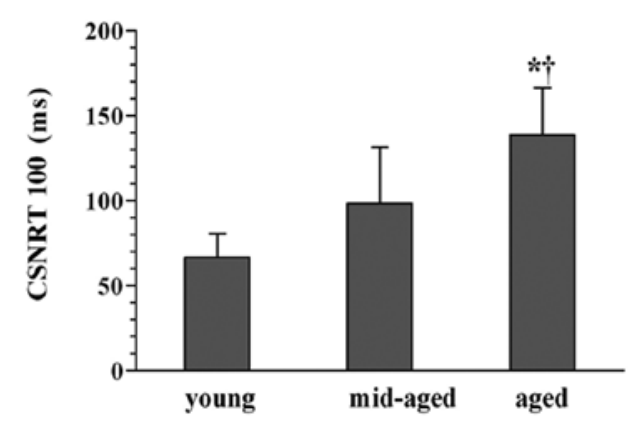

Figure 1. Global electrophysiology. (A) Typical representation of SNRT. (B) Comparison of P-wave duration (PWD) among the 3 groups. (C) Comparison of SNRT at a cycle length of $100 \mathrm{msec}$ in the different groups. (D) Comparison of CSNRT at a cycle length of $100 \mathrm{msec}$ in the different groups. A value of P<0.05 was considered to indicate a statistically significant difference. ${ }^{*} \mathrm{PWD}$ in the aged vs. the young group (P<0.05); ${ }^{\dagger} \mathrm{PWD}$ in the aged vs. the middle-aged group $(\mathrm{P}<0.05)$; SNRT, sinus node recovery period; CSNRT, corrected sinus node recovery period.

solution contained (in mmol/l) $137 \mathrm{NaCl}, 5.4 \mathrm{KCl}, 1.8 \mathrm{CaCl}_{2}$, $1 \mathrm{MgCl}_{2}, 0.33 \mathrm{NaH}_{2} \mathrm{PO}_{4}, 5$ HEPES, 10 D-glucose and 4-aminopyridine, $\mathrm{pH}$ 7.3-7.4 $(\mathrm{NaOH}) . \mathrm{Ca}^{2+}$ channels were blocked with $0.05 \mathrm{mmol} / 1 \mathrm{CdCl}_{2}$. Atropine of $1 \mu \mathrm{mol} / 1$ was used to prevent muscarinic receptor activation, and $5 \mu \mathrm{mol} / 1$ E-4031 was added to the external solution to block the rapid delayed rectifier current $\left(I_{k r}\right)$. In addition, EGTA was used for chelating free $\mathrm{Ca}^{2+}$, thereby blocking $I_{t o 2}$ and $I_{\mathrm{Na}-\mathrm{Ca}}$. For AP measurements, the internal solution contained (in mmol/l) $110 \mathrm{~K}$-aspartate, $20 \mathrm{KCl}, 1.8 \mathrm{MgCl}_{2}$, $10 \mathrm{HEPES}, 5 \mathrm{Mg}$-ATP,

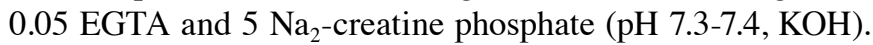
The external solution contained (in $\mathrm{mmol} / \mathrm{l}$ ) $137 \mathrm{NaCl}, 5.4 \mathrm{KCl}$, $1 \mathrm{MgCl}_{2}, 1.8 \mathrm{CaCl}_{2}, 10 \mathrm{D}$-glucose, $0.33 \mathrm{NaH}_{2} \mathrm{PO}_{4}$ and 5 HEPES (pH 7.3-7.4, NaOH). Signals were filtered with 2.9 and $10 \mathrm{kHz}$ Bessel filters and recorded by an Axopatch700 A amplifier with the Digidata1320A-pClamp 8.2 Data Acquisition System (Axon Instruments, Foster City, CA). All experiments were conducted at room temperature $\left(25 \pm 1^{\circ} \mathrm{C}\right)$.

Statistical analyses. Values are presented as the means \pm standard deviation. Surface ECG and esophageal electrogram were measured by 3 independent observers and compiled for statistical interpretation. Differences between groups were analyzed using a two-tailed Student's t-test, analysis of variance (ANOVA), Kruskal-Wallis H test, Mann-Whitney $\mathrm{U}$ test or Chi-square test, where appropriate. A two-tailed $\mathrm{P}<0.05$ was considered to indicate a statistically significant difference. All statistical analyses were performed with SPSS software.

\section{Results}

In vivo global electrophysiology. A total of 32 mice had the transesophageal rapid pacing procedure performed. No complications due to anesthesia or surgical preparation of the esophagus were observed in the young group and middle-aged group. Two out of 10 mice in the aged group died from either sinus bradycardia or complete atrioventricular block by rapid atrial pacing and had to be excluded from the analysis.

The threshold of pacing $(0.95 \pm 0.38 \mathrm{~mA})$ in the young group was almost compatible with the middle-aged group $(1.36 \pm 0.44 \mathrm{~mA})$ and the aged group $(1.22 \pm 0.21 \mathrm{~mA})$. There was no significant difference in SCL among the 3 groups [112.73 $\pm 17.78 \mathrm{sec}$ (young), 110.42 $\pm 11.64 \mathrm{sec}$ (middle-aged) and $117.34 \pm 23.73 \mathrm{sec}$ (aged)]. PWD in the aged group had a significantly higher prolongation than the young and middleaged group (Fig. 1B) $(\mathrm{P}<0.05)$. A typical representation of SNRT measurement is shown in Fig. 1A. SNRT and CSNRT at a S1S1 stimulation cycle length of $100 \mathrm{msec}$ in the aged group had a significantly higher prolongation relative to the young and middle-aged group $(\mathrm{P}<0.05)$; however, there was no significant difference between the young and middle-aged group (Fig. 1C and D) ( $\mathrm{P}>0.05)$. The inducibility of $\mathrm{AT} / \mathrm{F}$ among the 3 groups is shown in Table II. Although the duration of each AF episode was not significantly different among the groups, there was an overall induced rate of AT/F that increased significantly with age $(\mathrm{P}<0.05)$. Vulnerability of the atrial myocardium was defined as when $\mathrm{AT} / \mathrm{F}$ was successfully induced in the mouse model. 

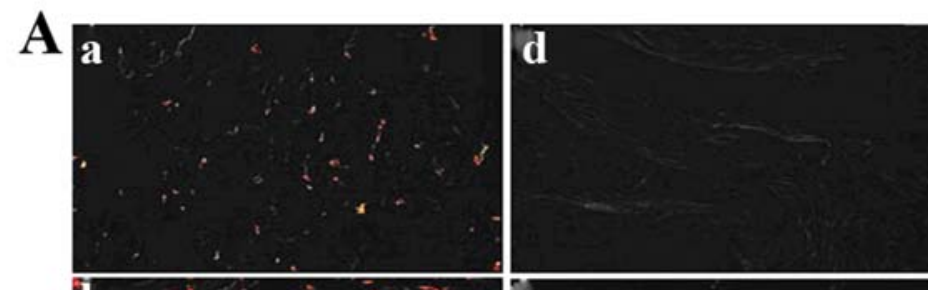

young
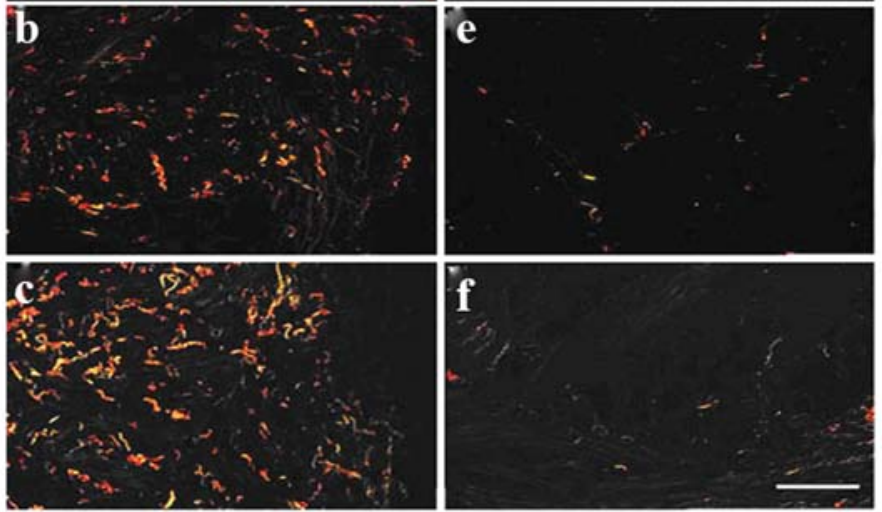

right atria

left atria

B

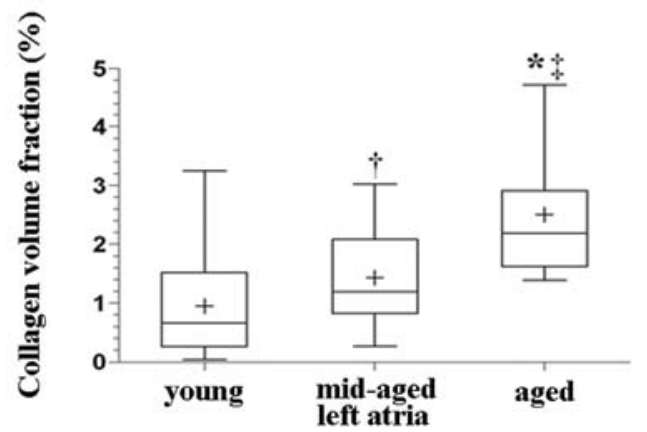

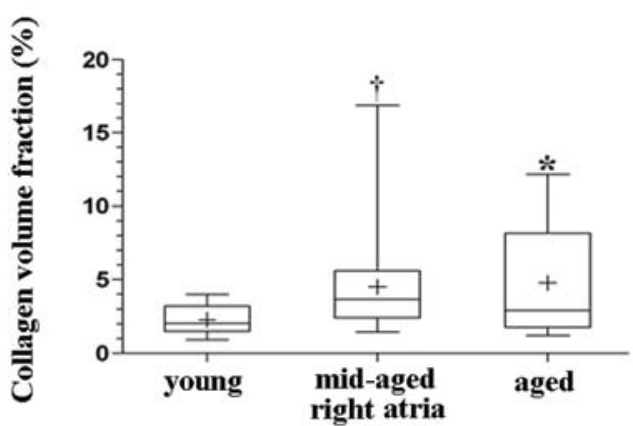

Figure 2. Collagen deposition in the left and right atrium. (A) Representative images of collagen deposition (x100-fold; scale bar, $100 \mu \mathrm{m}$ ). (B) Statistical comparisons shown by box-whisker plots. The data are separated into quartiles; the top of the box shows the 75th percentile, the line within the box shows the median, and the bottom of the box shows the 25th percentile. The top of the whisker denotes the maximum value, and the bottom of the whisker denotes the minimum value. + , median. ${ }^{*} \mathrm{P}<0.05$ represents the aged vs. the young group; ${ }^{{ }^{\circ}} \mathrm{P}<0.05$ represents the aged vs. the middle-aged group; ${ }^{\dagger} \mathrm{P}<0.05$ represents the middle-aged vs. the young group.

Age-related dynamic changes in the atrial CVF. Collagen fibers were much thicker in the right atrium than the left atrium, and the CVF was higher in the right atrium among the 3 groups (Fig. 2A). Spearman rank correlation analysis showed that the CVF in the left atrium significantly correlated with age $(\mathrm{P}<0.01, \mathrm{r}=0.592)$ (Fig. 2B). The $\mathrm{CVF}$ in the right atrium was higher in the aged and middle-aged groups than in the young group; however, but there was no significant difference between the aged and middle-aged groups. Spearman rank correlation analysis showed that the CVF in the right atrium correlated with age $(\mathrm{r}=0.326, \mathrm{P}<0.01)$; however, this did not achieve the level of the left atrium (Fig. 2B).

Overall, the right atrium showed significantly higher fibrosis than the left atrium in all the groups, and the extent of fibrosis in the left atrium had a higher positive correlation with age relative to the right atrium.

\section{Patch clamp experiments}

Age-related changes in Aps and $\mathrm{Cm}$. In the current clamp mode, APs were elicited by square current pulses of 400-600 pA amplitude and $3 \mathrm{msec}$ duration. A steady-state
AP was considered as the last of a train of 20 at the same stimulation rate. The APD at 20,50 and 90\% repolarization $\left(\mathrm{APD}_{20}, \mathrm{APD}_{50}, \mathrm{APD}_{90}\right.$, respectively) has a tendency towards prolonging followed by shortening in regard to the age development, whereas the $\mathrm{APD}_{90}$ in the left relative to the right atrium demonstrated a significant difference in the aged group $(\mathrm{P}<0.05)$ (Table III).

In the whole voltage clamp mode, single atrial myocyte $\mathrm{Cm}$ was elicited by a square pulse of depolarization. Mean left atrial $\mathrm{Cm}$ was $34.2 \pm 1.4 \mathrm{pF}$ (young) $(\mathrm{n}=33), 39.3 \pm 1.9 \mathrm{pF}$ (middle-aged) $(\mathrm{n}=33)$ and $46.7 \pm 2.4 \mathrm{pF}$ (aged) $(\mathrm{n}=19)$, respectively, while mean right atrial $\mathrm{Cm}$ was $30.0 \pm 1.4 \mathrm{pF}(\mathrm{n}=33)$, $32.8 \pm 1.9 \mathrm{pF}(\mathrm{n}=33)$ and $40.1 \pm 2.6 \mathrm{pF}(\mathrm{n}=19)$, respectively. These results demonstrate that $\mathrm{Cm}$ of the left and right atrium increases with advancing age, and there is a stronger tendency towards changes in left atrial $\mathrm{Cm}$ with age relative to right atrial Cm (Fig. 3).

Age-related changes of $I_{t o}$. Fig. $4 \mathrm{~A}$ shows voltage-dependent $I_{t o}$ elicited by voltage steps of $100 \mathrm{msec}$ to between -40 and +50 from $-50 \mathrm{mV}$. The holding potential of $-50 \mathrm{mV}$ was used to exclude possible $I_{N a}$ and $I_{C a . T}$ contamination during 
Table II. Comparisons of the inducibility of AT/F among the 3 groups.

\begin{tabular}{lccc}
\hline & Young & Middle-aged & Aged \\
\hline Induction rate of AF (\%) & 27.3 & $63.6^{\mathrm{a}}$ & $62.5^{\mathrm{b}}$ \\
Median duration for each AF (S) & $3.04 \pm 1.32$ & $2.54 \pm 0.86$ & $2.66 \pm 0.22$ \\
Induction rate of AT (\%) & 0 & $9.1^{\mathrm{a}}$ & $25^{\mathrm{b}}$ \\
Vulnerability to AT/F (\%) & 27.3 & $72.7^{\mathrm{a}}$ & $87.5^{\mathrm{b}}$ \\
\hline
\end{tabular}

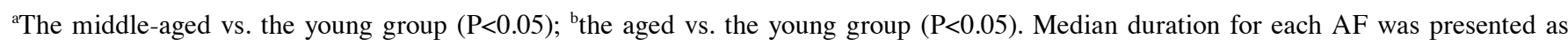
mean $\pm \mathrm{SD}$. AT/F, atrial tachycardia and fibrillation; AF, atrial fibrillation.

Table III. Comparison of APDs between the left and right atrium.

\begin{tabular}{|c|c|c|c|c|c|c|c|}
\hline \multirow[b]{2}{*}{ Group } & \multirow[b]{2}{*}{ No. } & \multicolumn{2}{|c|}{$\mathrm{APD}_{20}(\mathrm{msec})$} & \multicolumn{2}{|c|}{$\mathrm{APD}_{50}(\mathrm{msec})$} & \multicolumn{2}{|c|}{$\mathrm{APD}_{90}(\mathrm{msec})$} \\
\hline & & Left & Right & Left & Right & Left & Right \\
\hline Young & 7 & $3.9 \pm 0.2$ & $4.2 \pm 0.3$ & $5.0 \pm 0.6$ & $5.2 \pm 0.4$ & $22.1 \pm 2.6$ & $22.5 \pm 2.4$ \\
\hline Middle-aged & 7 & $5.5 \pm 0.5$ & $5.9 \pm 0.4$ & $9.3 \pm 1.0$ & $9.5 \pm 0.9$ & $31.9 \pm 3.2$ & $32.9 \pm 3.3$ \\
\hline Aged & 7 & $4.9 \pm 0.4$ & $5.4 \pm 0.6$ & $6.7 \pm 0.6$ & $7.5 \pm 0.9$ & $22.8 \pm 2.7$ & $27.6 \pm 2.8^{a}$ \\
\hline
\end{tabular}

${ }^{a} \mathrm{P}<0.05$ vs. the left atrium. Data are presented as the means \pm SD. APD, action potential duration.

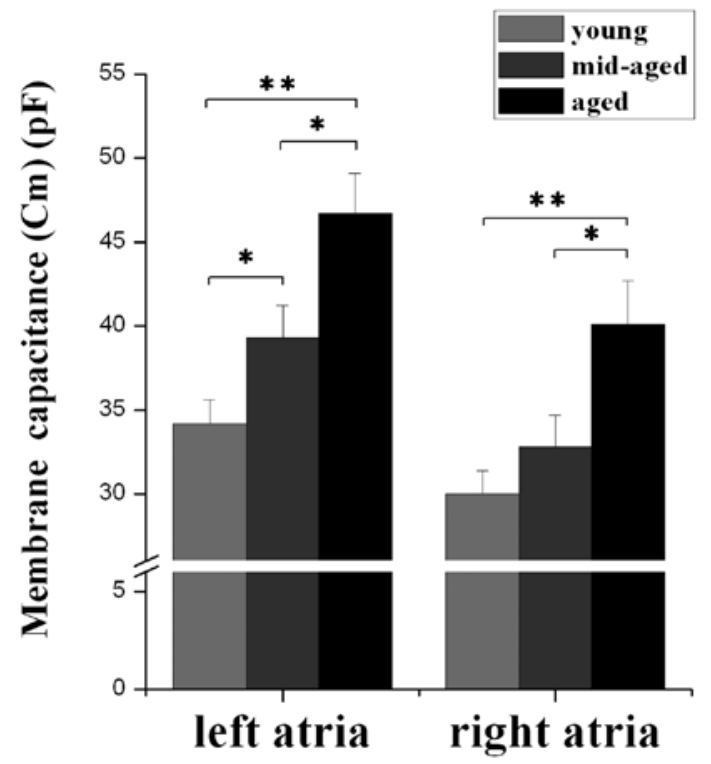

Figure 3. Membrane capacitance $(\mathrm{Cm})$. Comparison of atrial $\mathrm{Cm}$ among the 3 groups. ${ }^{*} \mathrm{P}<0.05,{ }^{* *} \mathrm{P}<0.01$.

current recording. We found that $I_{t o}$ was activated rapidly and achieved peak current within minutes after delivering testing potentials, followed by a rapid decrease in the steady state baseline current level. $I_{t o}$ increased with testing potentials in a typically voltage-dependent manner. When $4 \mathrm{mM} 4-\mathrm{AP}$ was added to the external solution, $I_{t o}$ was blocked by $>80 \%$ (Fig. 4A).

As shown in Fig. 4B, $I_{t o}$ was activated at $-30 \mathrm{mV}$ and current density was not significantly different among the 3 groups.
The current density of $I_{t o}$ in the aged group was larger than that in the young and middle-aged groups at $-10 \mathrm{mV}(\mathrm{P}<0.05)$, and this was even more significant when the testing potential was increased. At a test potential of $+50 \mathrm{mV}$, the current density was $7.8 \pm 0.9 \mathrm{pA} / \mathrm{pF}(\mathrm{n}=6), 10.0 \pm 1.0 \mathrm{pA} / \mathrm{pF}(\mathrm{n}=6)$ and $12.0 \pm 1.4 \mathrm{pA} / \mathrm{pF}(\mathrm{n}=5)$ in the young, middle-aged and aged groups, respectively, indicating that current density increased with age $(\mathrm{P}<0.05)$.

Fig. 4C shows the activation curve of $I_{t o}$ by depolarization to +50 from $-50 \mathrm{mV}$. With age, the voltage-dependent $I_{t o}$ activation curve slightly shifted in a negative direction. $V_{1 / 2}$ and $K$ were $12.8 \pm 0.9$ and $9.4 \pm 0.9 \mathrm{mV}(\mathrm{n}=6), 11.5 \pm 0.6$ and $9.5 \pm 0.6 \mathrm{mV}(\mathrm{n}=6)$, and $10.9 \pm 0.8$ and $9.2 \pm 0.7 \mathrm{mV}(\mathrm{n}=6)$ in the young, middle-aged and aged groups, respectively, indicating that there were no significant changes in $V_{1 / 2}$ and $K$ with age $(\mathrm{P}>0.05)$.

The voltage dependence of the steady state inactivation relationship was investigated using a standard 2-pulse protocol: a 2,000 $\mathrm{msec}$ preconditioning pulse ranging from -80 to $0 \mathrm{mV}$ followed by a $140 \mathrm{msec}$ test pulse to $+50 \mathrm{mV}$. $V_{1 / 2}$ and $K$ were $-49.7 \pm 2.1$ and $8.1 \pm 1.9 \mathrm{mV}(\mathrm{n}=6),-48.8 \pm 1.6$ and $7.3 \pm 1.5 \mathrm{mV}(\mathrm{n}=6)$, and $-48.7 \pm 1.5$ and $7.0 \pm 1.4 \mathrm{mV}(\mathrm{n}=6)$ in the young, middle-aged and aged groups, respectively, indicating that there were no significant changes in $V_{1 / 2}$ and $K$ with age (P>0.05) (Fig. 4D).

Age-related changes of $I_{k u r}$. $I_{k u r}$ were elicited by a $80-\mathrm{msec}$ prepulse to $+30 \mathrm{mV}$ to inactivate $I_{t o}$, followed by 140 -msec test pulses between -40 and $+50 \mathrm{mV}$ after a 10 -msec interval from $-50 \mathrm{mV}$ at $0.2 \mathrm{~Hz}$. At a test potential of $+50 \mathrm{mV}$, the current density was $4.1 \pm 0.7 \mathrm{pA} / \mathrm{pF}(\mathrm{n}=6), 4.2 \pm 1.0 \mathrm{pA} / \mathrm{pF}(\mathrm{n}=6)$ and $4.2 \pm 0.8 \mathrm{pA} / \mathrm{pF}(\mathrm{n}=5)$ in the young, middle-aged and aged group, respectively, indicating that there was no significant difference among the groups $(\mathrm{P}>0.05)$ (Fig. 5B). 

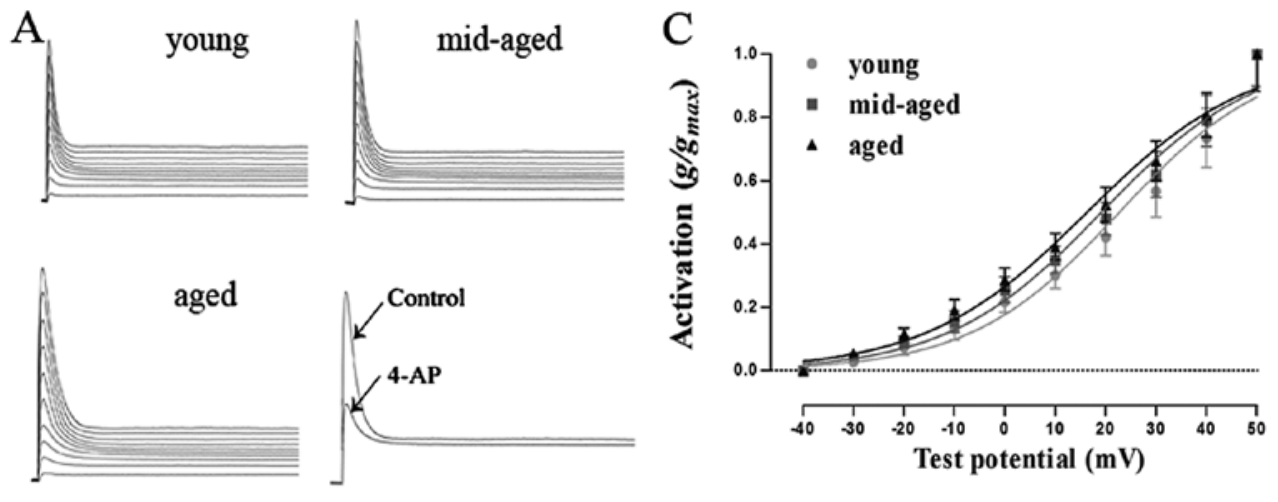

B

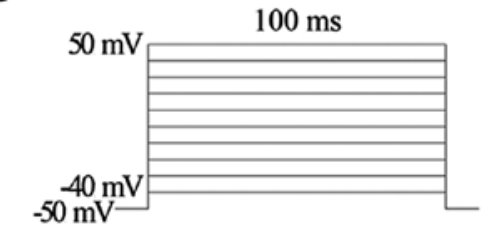
$\rightarrow$ young
$\rightarrow$ mid-aged
$\leftarrow$ aged

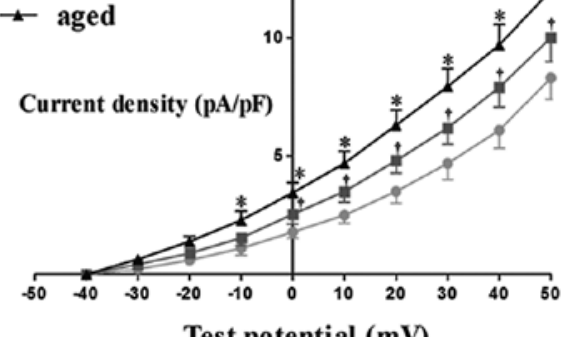

Test potential (mV)
$\mathrm{D}$
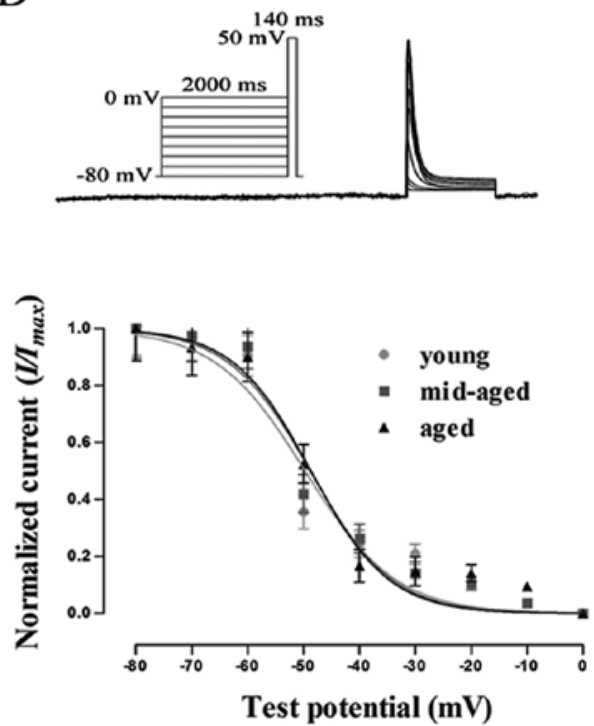

Figure 4. Analyses of $I_{t o}$ currents. (A) Original tracings of $I_{t o}$ in atrial myocytes in the different groups (young, middle-aged and aged). Original tracings of $I_{t o}$ before and after addition of 4-AP are shown. (B) I-V relationship curve of $I_{t o}$ in the different groups. The stimulation protocol of $I_{t o}$ is shown. (C) Activation curves of $I_{t o}$ from atrial myocytes in the different groups. (D) Inactivation curves of $I_{t o}$. Typical original tracings of inactivation curves are shown. " $\mathrm{P}<0.05$ represents the aged vs. the middle-aged and young groups; ${ }^{\dagger} \mathrm{P}<0.05$ represents the middle-aged vs. the young groups.

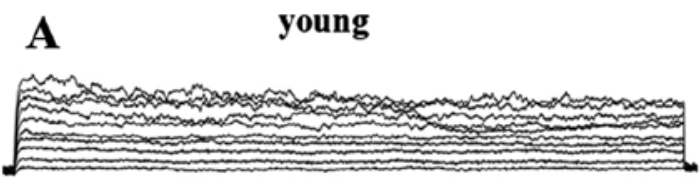

mid-aged
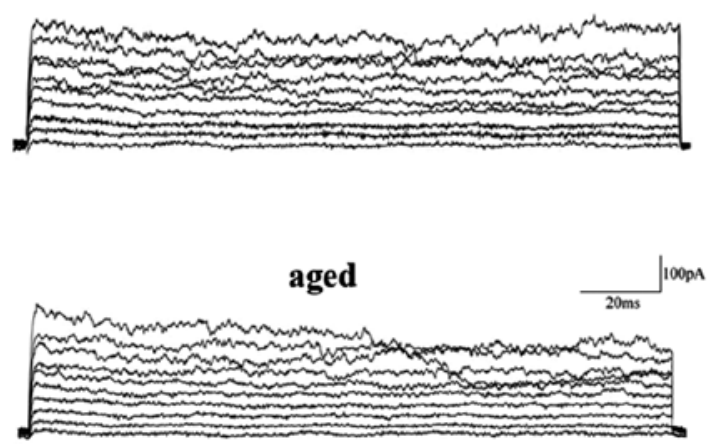

B
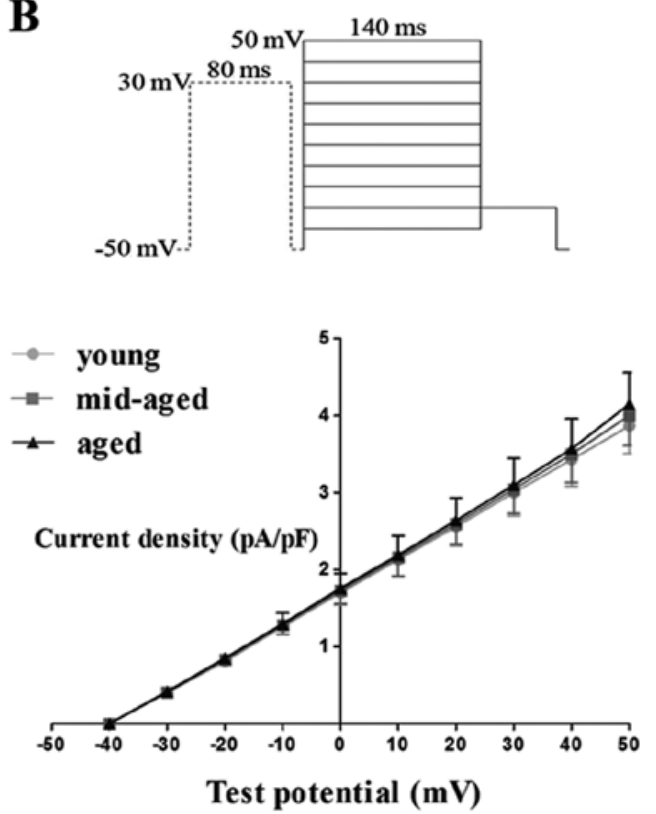

Figure 5. Analyses of $I_{k u r}$ currents. (A) Original tracings of $I_{k u r}$ in atrial myocytes in the different groups (young, middle-aged and aged). (B) Stimulation protocol of $I_{k u r}$. The I-V relationship curves of $I_{k u r}$ from atrial myocytes in the different groups are shown. 


\section{Discussion}

Main findings. In the present study, we performed measurements of in vivo global electrical properties by the transesophageal atrial rapid pacing method and found the following: (i Compared with the other 2 groups, PWD, as well as the SNRT and CSNRT at a cycle length of $100 \mathrm{msec}$ were longer in the aged group, indicating the slowing of intra-atrial conduction and sinus nodal dysfunction. ii) Although the duration of each AF episode was not significantly different among the groups, the total inducibility, represented as the key point for evaluating vulnerability to $\mathrm{AT} / \mathrm{F}$, was significantly increased with aging. As regards changes in collagen in the left and right atrium, we found that the right atrium showed significantly higher fibrosis relative to the left atrium in all the groups, whereas the extent of fibrosis in the left atrium had a higher positive correlation with age compared to the right atrium. Furthermore, from the cellular electrophysiological experiments, the results showed that age-related augmented $I_{t o}$ currents and no age-related changed $I_{k u r}$ currents, as well as the subsequent AP discrepancy in old age, contributed to the dispersion of repolarization, which further promoted AF.

Age-related abnormal pulse initiation. Abnormal pulse initiation due to the sinoatrial node dysfunction is associated with advancing age (17). If the sinus node fails to fire, then there is a significant tendency towards manifestation of abnormal impulse initiation in atrial cells. In the present study, measurements of SNRT and CSNRT at a cycle length of $100 \mathrm{msec}$ showed that aged mice had a longer SNRT than the young and middle-aged mice. Recently, Stiles et al (18) demonstrated that the prolongation of SNRT, regarded as sinus node dysfunction, is a key contributor which facilitates the progression of paroxysmal AF to permanent AF. Surprisingly, it has been previously found that although the SCL increases with age, the SNRT decreases with age (19), which is in contrast to our results. The likely mechanism for these observations is that conduction in the sinus node worsens with age and limits the ability of an extra-stimulus to overdrive and suppress sinus node automaticity. A previous study found enlargement of the sinus node, hypertrophy of sinus nodal cells, and remodeling of the extracellular matrix in aged rats (20). Therefore, they concluded that the age-dependent decrease in sinus nodal function is due to structural remodeling of the sinus node. Consequently, decreased automaticity and conductivity of the sinus node are associated with shortening of atrial refractoriness and increasing the atrial effective refractory period (ERP) dispersion, which in turn creates a substrate for AF initiation and perpetuation (21).

Age-related abnormal pulse conduction. Abnormal pulse conduction in the aged atrium is a contributor to the maintenance of AF. Hence, it is important to comprehend the nature of both the electrical and structural remodeling that occurs with age. In the current study, surface ECG showed that PWD was significantly longer in the aged group than in the young group and had a prolongation tendency in the middle-aged group, which indicated that the intra-atrial pulse conduction time was prolonged with increasing age. It has been reported that the PWD significantly correlates with age in animals (6) and humans (22), and depends mainly on intra-atrial conduction time and atrial size. The slowing of intra-atrial conduction, one of the most important requirements for the initiation of re-entry, is more frequently observed in patients with paroxysmal AF than in normal subjects (23). Moreover, atrial dilatation increases the amount of atrial tissue that can accommodate multiple re-entry circuits (3). Therefore, P-wave prolongation and atrial dilatation possibly reflect age-related atrial remodeling that is favorable for AF; thus, aged mice, compared to young mice, possess a substrate that is more susceptible to AF.

It is well known that the profound structural remodeling that occurs with age affects the ability of a premature beat to penetrate the substrate and initiate propagation. In the present study, we observed a significant correlation between the degree of interstitial fibrosis and aging in the left and right atrium. In a previous study, Burkauskiene et al (24) demonstrated that the diameter and area of collagen fibers positively correlate with aging, whereas the amount of collagen fibers does not alter with age. This indicates that changes in the collagen network may be a progressively developing process. Another study reported that spatial distribution of the collagen network was significantly different in patients with ischemic cardiomyopathy compared with those without heart disease (25). Taken together, these findings, including ours, suggest that atrial interstitial fibrosis, which is increased with age and heart disease, possibly enhances the heterogeneous slowing of atrial conduction, which may be responsible for the age-related increase in AF vulnerability. In general, there is a close correlation between the onset of $\mathrm{AF}$ and structural remodeling in the left atrium (26). However, Nakajima et al (27) found that although similar levels of cardiomyocyte apoptosis were present in the right and left atria of MHC-TGFcys ${ }^{33}$ ser hearts, the extent of fibrosis was more pronounced in the right atrium than in the left atrium. They also revealed cardiomyocyte cell activity in left atrial cardiomyocytes, but not in right atrial cardiomyocytes by tritiated thymidine incorporation studies. These data support the notion that cardiomyocyte cell cycle induction can antagonize fibrosis in the myocardium. In agreement with previous results, the present study found that the collagen volume of the right atrium was higher than that in the left atrium in all the groups. Although we found that the volume of fibers increased with age in the left and right atrium, less fibrosis was present in the left atrium, which may be due to faster cell cycle activity in the left atrium.

Age and predisposition to $A F$. In our study, we compared the inducibility of atrial AT/F to evaluate vulnerability to arrhythmias. Our results showed that the inducibility of atrial AT/F significantly increased with age, indicating that aging is a key contributor to AF episodes. In addition, inducible AF was also observed in the young and middle-aged group, in contrast to the negative results presented in the study by Guzadhur et al (28). A possible reason is that the susceptibility of the mouse atrium to the induction of AF appears to be increased when transesophageal pacing instead of isolated pacing (28) or transvenous pacing (29) is performed. It is possible that relative to isolated or transvenous stimulation, unipolar transesophageal atrial stimulation affects a larger area of the atrium, which may involve the vagal nerve. This may result in a more global inho- 
mogeneous and abnormally delayed activation of the atrium, which is believed to be the basic mechanism of induction of atrial arrhythmia by premature depolarization. Moreover, the atrial stretch is known to enhance atrial vulnerability and may be augmented in this setting of transesophageal stimulation. Of note, the present study showed that PWD was only prolonged in aged group relative to other 2 groups, not reconciling the high incidence of tachyarrhythmia in the middle-aged and aged group. The discrepancy may be due to more electrophysiological remodeling in the middle-aged group, while structural remodeling was predominant in the aged group.

Age-related cellular electrophysiological changes conducive to $A F$. AP changes with age are affected by different types of inward and outward ion currents. $I_{t o}$ and $I_{k u r}$, which are the major ion currents involved in the repolarization process in mice, contribute to age-related AP changes and AF initiation and maintenance. In the present study, a $\mathrm{Ca}^{2+}$-independent transient outward current $\left(I_{t o l}\right)$ was recorded by the addition of $\mathrm{CdCl}_{2}$ to the external solution to block the L-type $\mathrm{Ca}^{2+}$ current and the addition of a $\mathrm{Ca}^{2+}$ chelating agent (EGTA) to the internal solution. Our results showed that $I_{t o}$ was suppressed by $>80 \%$ after the addition of 4-AP. Dun et al (30) found that $I_{t o}$ current densities of canine right atria were significantly increased in the aged group compared with the adult group, whereas inactivation slowing and steady state inactivation curves showed a trend to shift toward depolarization. Mansourati et al (31) also found that $I_{\text {to }}$ was decreased in adult patients with heart diseases; however, the results did not reflect the normal electrophysiological properties of humans due to the existence of pathological factors. We found that $I_{t o}$ current densities increased with age, which is in agreement with the results presented in the study by Crumb et al (32), whereas the activation and inactivation properties of $I_{t o}$ were not different among the 3 groups. As such, the augmentation of $I_{t o}$ may have contributed to the shortening of atrial APs in the aged group. Following the inactivation of $I_{t o l}$, a slowly inactivating current remains, termed $I_{\text {sus }}$ or $I_{k u r}$. There is a general consensus that the $\mathrm{K}_{\mathrm{V}} 1.5$ subunit is largely responsible for $I_{k u r}$ in human atrial myocytes, similar to other species, such as the mouse and dog. Since this subunit is much less abundant in the ventricle than in the atrial myocardium, it appears to be quite specific to atrial myocytes. $I_{k u r}$ densities are significantly increased in aged canine right atrial cells compared with adult cells (30). However, KN-93, a specific CaMKII inhibitor, has been shown to dramatically inhibit $I_{k u r}$ in aged right atrial cells compared with adult right atrial cells, indicating that increased $I_{k u r}$ in aged RA cells is most likely due to CaMKII upregulation (33). To the best of our knowledge, no age-related changes in $I_{k u r}$ have thus far been observed in human atrial myocytes. In alignment with human atrial data, the present study using mice also demonstrated that there were no age-related changes in $I_{k u r}$ among the different groups, which may be due to stability after birth.

The abnormal shape of the AP contour derived from a single isolated cell is conducive to AF. Anyukhovsky et al (34) found that $\mathrm{APD}_{90}$ averaged across all regions was significantly longer in the aged compared with adult tissues. Notably, the range of $\mathrm{APD}_{90}$ values was wider in the aged vs. the adult group as a result of an increase in duration, occurring mainly in right atrial fibers (34). However, clear-cut evidence of age-related changes in APs in normal human atria is lacking, as no agerelated changes in APD and ERP have been obtained (35). In the present study, we found that $\mathrm{APD}_{90}$ in the left and right atrium was prolonged with age, followed by a decrease in the aged group, particularly in the left atrium. In agreement with our report, Brorson et al (36) used 4 age groups to examine right atrial ERPs and monophasic APs in healthy male humans in vivo. They found that the middle-aged group showed a significantly longer right atrial ERP than the other groups. However, there was no progressive trend in APD with age. Thus, these results do not support the traditional view that APDs are prolonged with age. In general, AP is prolonged followed by shortening with age may due to a decrease in the calcium current in aged atrial tissue (6).

Limitations. The transesophageal stimulation technique used in the current study includes difficulties in atrial electrogram detection during AF, and therefore, the evaluation of the atrial AF heart rate is not always possible. Additionally, due to the limitations of experimental devices and technologies, measurements of atrial ERP and intra-atrial conduction velocity were not conducted for further insight into the electrophysiological mechanisms. Finally, it should be acknowledged that the pathophysiological characteristics of AF in mice may be different from those in humans.

In conclusion, the results from our study demonstrate that aging causes structural remodeling, as well as integrative and cellular electrophysiological changes, facilitating increased dispersion of repolarization and re-entry formation. This in turn creates a substrate for AF initiation and perpetuation. Furthermore, there may be an association between structural and electrical remodeling, which can exert vicious cycle effects on the development of AF.

\section{Acknowledgements}

The present study was supported by the Tianjin Municipal Science and Technology Committee (09ZCZDSF04200 and 11JCYBJC12000) and the Natural Science Foundation of China (81170238 and 81070121).

\section{References}

1. Fuster V, Ryden LE, Cannom DS, et al: 2011 ACCF/AHA/HRS focused updates incorporated into the ACC/AHA/ESC 2006 guidelines for the management of patients with atrial fibrillation: a report of the American College of Cardiology Foundation/ American Heart Association Task Force on practice guidelines. Circulation 123: e269-e367, 2011.

2. Jalife J: Deja vu in the theories of atrial fibrillation dynamics. Cardiovasc Res 89: 766-775, 2011.

3. Nattel S, Burstein B and Dobrev D: Atrial remodeling and atrial fibrillation: mechanisms and implications. Circ Arrhythm Electrophysiol 1: 62-73, 2008.

4. Spach MS, Miller WT III, Dolber PC, Kootsey JM, Sommer JR and Mosher CE Jr: The functional role of structural complexities in the propagation of depolarization in the atrium of the dog. Cardiac conduction disturbances due to discontinuities of effective axial resistivity. Circ Res 50: 175-191, 1982.

5. Koura T, Hara M, Takeuchi S, et al: Anisotropic conduction properties in canine atria analyzed by high-resolution optical mapping: preferential direction of conduction block changes from longitudinal to transverse with increasing age. Circulation 105: 2092-2098, 2002. 
6. Anyukhovsky EP, Sosunov EA, Plotnikov A, et al: Cellular electrophysiologic properties of old canine atria provide a substrate for arrhythmogenesis. Cardiovasc Res 54: 462-469, 2002.

7. Wongcharoen W, Chen YC, Chen YJ, et al: Aging increases pulmonary veins arrhythmogenesis and susceptibility to calcium regulation agents. Heart Rhythm 4: 1338-1349, 2007.

8. Wongcharoen W, Chen YC, Chen YJ, Lin CI and Chen SA: Effects of aging and ouabain on left atrial arrhythmogenicity. J Cardiovasc Electrophysiol 18: 526-531, 2007.

9. Hayashi H, Wang C, Miyauchi Y, et al: Aging-related increase to inducible atrial fibrillation in the rat model. J Cardiovasc Electrophysiol 13: 801-808, 2002.

10. Xu D, Murakoshi N, Tada H, Igarashi M, Sekiguchi Y and Aonuma K: Age-related increase in atrial fibrillation induced by transvenous catheter-based atrial burst pacing: an in vivo rat model of inducible atrial fibrillation. J Cardiovasc Electrophysiol 21: 88-93, 2010.

11. Guzadhur L, Jiang W, Pearcey SM, et al: The age-dependence of atrial arrhythmogenicity in $\mathrm{Scn} 5 \mathrm{a}^{+/-}$murine hearts reflects alterations in action potential propagation and recovery. Clin Exp Pharmacol Physiol 39: 518-527, 2012.

12. Vaidya D, Morley GE, Samie FH and Jalife J: Reentry and fibrillation in the mouse heart. A challenge to the critical mass hypothesis. Circ Res 85: 174-181, 1999.

13. Fox JG, Davisson MT, Quimby FW, Barthold SW, Newcomer CE and Smith AL (eds): The Mouse in Biomedical Research. Normative Biology, Husbandry, and Models. Vol. 3, 2nd edition. Elsevier, London, 2007.

14. Schrickel JW, Bielik H, Yang A, et al: Induction of atrial fibrillation in mice by rapid transesophageal atrial pacing. Basic Res Cardiol 97: 452-460, 2002.

15. Zhou X, Yun JL, Han ZQ, et al: Postinfarction healing dynamics in the mechanically unloaded rat left ventricle. Am J Physiol Heart Circ Physiol 300: H1863-H1874, 2011.

16. Wolska BM and Solaro RJ: Method for isolation of adult mouse cardiac myocytes for studies of contraction and microfluorimetry. Am J Physiol 271: H1250-H1255, 1996.

17. Lakatta EG and Sollott SJ: The 'heartbreak' of older age. Mol Interv 2: 431-446, 2002

18. Stiles MK, John B, Wong CX, et al: Paroxysmal lone atrial fibrillation is associated with an abnormal atrial substrate: characterizing the 'second factor'. J Am Coll Cardiol 53: 1182-1191, 2009.

19. Taneja T, Mahnert BW, Passman R, Goldberger J and Kadish A: Effects of sex and age on electrocardiographic and cardiac electrophysiological properties in adults. Pacing Clin Electrophysiol 24: 16-21, 2001.

20. Yanni J, Tellez JO, Sutyagin PV, Boyett MR and Dobrzynski H: Structural remodelling of the sinoatrial node in obese old rats. J Mol Cell Cardiol 48: 653-662, 2010

21. Li G, Liu E, Liu T, et al: Atrial electrical remodeling in a canine model of sinus node dysfunction. Int J Cardiol 146: 32-36, 2011.
22. Kojodjojo P, Kanagaratnam P, Markides V, Davies DW and Peters N: Age-related changes in human left and right atrial conduction. J Cardiovasc Electrophysiol 17: 120-127, 2006.

23. Kistler PM, Sanders P, Fynn SP, et al: Electrophysiologic and electroanatomic changes in the human atrium associated with age. J Am Coll Cardiol 44: 109-116, 2004.

24. Burkauskiene A, Mackiewicz Z, Virtanen I and Konttinen YT: Age-related changes in myocardial nerve and collagen networks of the auricle of the right atrium. Acta Cardiol 61: 513-518, 2006.

25. Burkauskiene A: Age-related changes in the structure of myocardial collagen network of auricle of the right atrium in healthy persons and ischemic heart disease patients. Medicina (Kaunas) 41: 145-154, 2005

26. Abhayaratna WP, Seward JB, Appleton CP, et al: Left atrial size: physiologic determinants and clinical applications. J Am Coll Cardiol 47: 2357-2363, 2006.

27. Nakajima H, Nakajima HO, Dembowsky K, Pasumarthi KB and Field LJ: Cardiomyocyte cell cycle activation ameliorates fibrosis in the atrium. Circ Res 98: 141-148, 2006.

28. Guzadhur L, Pearcey SM, Duehmke RM, et al: Atrial arrhythmogenicity in aged Scn $5 \mathrm{a}^{+} /$DeltaKPQ mice modeling long QT type 3 syndrome and its relationship to $\mathrm{Na}^{+}$channel expression and cardiac conduction. Pflugers Arch 460: 593-601, 2010.

29. Wakimoto H, Maguire CT, Kovoor P, et al: Induction of atrial tachycardia and fibrillation in the mouse heart. Cardiovasc Res 50: 463-473, 2001.

30. Dun W, Yagi T, Rosen MR and Boyden PA: Calcium and potassium currents in cells from adult and aged canine right atria. Cardiovasc Res 58: 526-534, 2003.

31. Mansourati J and Le Grand B: Transient outward current in young and adult diseased human atria. Am J Physiol 265: H1466-H1470, 1993.

32. Crumb WJ Jr, Pigott JD and Clarkson CW: Comparison of Ito in young and adult human atrial myocytes: evidence for developmental changes. Am J Physiol 268: H1335-H1342, 1995.

33. Dun W, Chandra P, Danilo P, Rosen MR and Boyden PA: Chronic atrial fibrillation does not further decrease outward currents. It increases them. Am J Physiol Heart Circ Physiol 285: H1378-H1384, 2003.

34. Anyukhovsky EP, Sosunov EA, Chandra P, et al: Age-associated changes in electrophysiologic remodeling: a potential contributor to initiation of atrial fibrillation. Cardiovasc Res 66: 353-363, 2005.

35. Spach MS, Dolber PC and Anderson PA: Multiple regional differences in cellular properties that regulate repolarization and contraction in the right atrium of adult and newborn dogs. Circ Res 65: 1594-1611, 1989.

36. Brorson L and Olsson SB: Right atrial monophasic action potential in healthy males. Studies during spontaneous sinus rhythm and atrial pacing. Acta Med Scand 199: 433-446, 1976. 